Computer Vision, Graphics, and Pattern Recognition Group Department of Mathematics and Computer Science

University of Mannheim

D-68131 Mannheim, Germany

\author{
Reihe Informatik \\ $3 / 2000$ \\ Efficient Image Segmentation Using \\ Partial Differential Equations and Morphology
}

Joachim Weickert

Technical Report 3/2000

Computer Science Series

February 2000

This report is a revision of the DIKU Report No. 98/10, Department of Computer Science, University of Copenhagen, Denmark, May 1998.

The technical reports of the CVGPR Group are listed at the site http://www.ti. uni-mannheim/ bmg/Publications-e.html 


\title{
Efficient Image Segmentation Using Partial Differential Equations and Morphology
}

\author{
Joachim Weickert \\ Computer Vision, Graphics, and Pattern Recognition Group, \\ Department of Mathematics and Computer Science, \\ University of Mannheim, D-68131 Mannheim, Germany \\ E-mail: Joachim.Weickert@ti.uni-mannheim.de \\ WWW: http://www.ti.uni-mannheim.de/ bmg/weickert/
}

\begin{abstract}
The goal of this paper is to investigate segmentation methods that combine fast preprocessing algorithms using partial differential equations (PDEs) with a watershed transformation with region merging. We consider two well-founded PDE methods: a nonlinear isotropic diffusion filter that permits edge enhancement, and a convex nonquadratic variational image restoration method which gives good denoising. For the diffusion filter, an efficient algorithm is applied using an additive operator splitting (AOS) that leads to recursive and separable filters. For the variational restoration method, a novel algorithm is developed that uses AOS schemes within a Gaussian pyramid decomposition. Examples demonstrate that preprocessing by these PDE techniques significantly improves the watershed segmentation, and that the resulting segmentation method gives better results than some traditional techniques. The algorithm has linear complexity and it can be used for arbitrary dimensional data sets. The typical CPU time for segmenting a $256^{2}$ image on a modern PC is far below one second.
\end{abstract}

Keywords: nonlinear diffusion, variational methods, image restoration, additive operator splitting, Gaussian pyramid, watershed segmentation, region merging

CR Subject Classification: I.4.6, I.4.3, I.4.4.

\section{Introduction}

Segmentation is one of the bottlenecks of many image analysis and computer vision tasks ranging from medical image processing to robot navigation. Ideally it should be efficient to compute and correspond well with the physical objects depicted in the image. This also requires that segmentation gives a complete partitioning of the image such that object contours are closed and no dangling edges exist.

In the last decade much research on PDE-based regularization methods has been carried out; see e.g. $[1,2,3,4]$ for recent overviews. Although the promising results suggest that they might be attractive as a preprocessing step for many subsequent image analysis methods, little research has actually been carried out which combines 
PDE-based preprocessing methods with other techniques. One of the problems was that PDE-based methods have been considered as being too slow in order to become an adequate partner for efficient other methods. This shows the need to further develop efficient algorithms for PDE-based techniques.

The goal of the present paper is to address these topics in the following way:

- Image segmentation is achieved by means of a watershed algorithm. This popular morphological method is more more than an edge detector: it gives a true image partitioning without dangling edges. The watershed segmentation is sufficiently fast for most applications, but it suffers from the limitation that many irrelevant minima cause an oversegmentation.

- In order to reduce the oversegmentation problem we study the use of two PDEbased techniques for preprocessing the image before segmentation: the nonlinear diffusion technique by Catté et al. [5] which allows edge enhancement, and a nonquadratic variational restoration technique of Schnörr [6] and Charbonnier et al. [7] which is well-suited for edge-preserving image denoising. These methods have been chosen as simple prototypes of PDEs that are mathematically wellfounded: they are well-posed in the sense of Hadamard in that they have a unique solution which is stable with respect to perturbations of the original image.

- As an efficient algorithm for the nonlinear diffusion filter we apply a recently developed method based on an additive operator splitting (AOS) [8]. It leads to separable and recursive filters. For the nonquadratic variational image restoration method, we develop a novel algorithm: minimization of the energy functional is achieved by considering a steepest descent method that leads to a diffusionreaction PDE. This $\mathrm{PDE}$ is then solved by a modified AOS algorithm that is embedded in a Gaussian pyramid decomposition.

The resulting segmentation algorithms can be generalized in a straightforward way to arbitrary dimensional data sets. Their complexity is linear in the pixel number, and they produce identical results when the image is rotated by 90 degrees. An overall CPU time of less than one second for segmenting a $256^{2}$ image on a typical PC or workstation makes them attractive for many time-critical applications.

The paper is organized as follows. Section 2 sketches the basic structure of the contrast-enhancing nonlinear diffusion filter and the convex nonquadratic restoration method. In Section 3 we discuss efficient and reliable numerical techniques for these methods. They are based on an additive operator splitting. For approximating the variational restoration method, these AOS techniques are extended to novel pyramid AOS schemes. In Section 4 we describe the watershed algorithm with region merging, and in Section 5 we illustrate the usefulness of the combined segmentation process by applying it to several examples and comparing it with classic approaches. The paper is concluded with a summary in Section 6 .

Related work. The work presented here has been influenced by several related approaches. Closest in terms of efficient PDE-based regularization methods is the work 
of Acton [9] on multigrid versions for nonlinear diffusion filtering. They are, however not based on AOS schemes and they do not use methods with a reaction term. It is common to supplement watershed segmentations with tools for reducing the oversegmentation problem. An algorithm by Orphanoudakis et al. [10] also uses region merging for this purpose, but it applies statistical instead of PDE-based smoothing strategies. Promising results of combining watershed algorithms with nonlinear diffusion have been described recently by De Vleeschauer et al. [11] and Sijbers et al. [12]. Investigations of watershed algorithms within scale-space hierarchies have been carried out by Griffin et al. [13], Olsen [14], Sramek and Wrbka [15], and Olsen and Sporring [16] for the linear diffusion scale-space, and by Jackway [17] for the dilation-erosion scale-space. A nonmorphological segmentation algorithm based on nonlinear diffusion scale-spaces has been studied by Niessen et al. $[18,19]$. This discussion shows that the novelty of our approach consist of developing pyramid AOS algorithms for efficient PDE-based regularization, and combining fast AOS-based algorithms with an important morphological segmentation tool, the watershed algorithm. This results in a fast segmentation method. A preliminary version of the present manuscript has been presented at a conference [20].

\section{PDE-Based Regularization}

Below two prototypes for well-posed PDE-based regularization techniques are presented. The first one allows contrast enhancement, while the second one can be expressed as an energy minimization method. These two methods are only representatives of a much larger class of diffusion-based smoothing methods. For a more detailed treatment of this topic the reader is referred to [4].

\subsection{The Nonlinear Diffusion Filter of Catté et al.}

In the $m$-dimensional case the filter of Catté, Lions, Morel and Coll [5] has the following structure:

Let $\Omega:=\left(0, a_{1}\right) \times \ldots \times\left(0, a_{m}\right)$ be our image domain and consider a (scalar) image $f(x)$ as a bounded mapping from $\Omega$ into the real numbers $\mathbb{R}$. Then a filtered image $u(x, t)$ of $f(x)$ is calculated by solving the diffusion equation with the original image as initial state, and reflecting boundary conditions:

$$
\begin{aligned}
& \partial_{t} u=\operatorname{div}\left(g\left(\left|\nabla u_{\sigma}\right|^{2}\right) \nabla u\right) \\
& u(x, 0)=f(x), \\
& \left.\partial_{n} u\right|_{\partial \Omega}=0,
\end{aligned}
$$

where $n$ denotes the normal to the image boundary $\partial \Omega$.

The "time" $t$ is a scale parameter: larger values lead to simpler image representations. In order to reduce smoothing at edges, the diffusivity $g$ is chosen as a decreasing function of the edge detector $\left|\nabla u_{\sigma}\right|^{2}$, where $\nabla u_{\sigma}$ is the gradient of a Gaussian-smoothed version 
of $u$ :

$$
\begin{aligned}
\nabla u_{\sigma} & :=\nabla\left(K_{\sigma} * u\right) \\
K_{\sigma} & :=\frac{1}{\left(2 \pi \sigma^{2}\right)^{m / 2}} \exp \left(-\frac{|x|^{2}}{2 \sigma^{2}}\right)
\end{aligned}
$$

We use the diffusivity

$$
g\left(s^{2}\right):= \begin{cases}1 & \left(s^{2}=0\right) \\ 1-\exp \left(\frac{-3.315}{(s / \lambda)^{8}}\right) & \left(s^{2}>0\right) .\end{cases}
$$

For such rapidly decreasing diffusivities, smoothing on both sides of an edge is much stronger than smoothing across it. This selective smoothing process prefers intraregional smoothing to interregional blurring. The factor 3.315 ensures that the flux $\Phi(s):=$ $s g\left(s^{2}\right)$ is increasing for $|s| \leq \lambda$ and decreasing for $|s|>\lambda$. Thus, $\lambda$ is a contrast parameter separating low-contrast regions with (smoothing) forward diffusion from high-contrast locations where backward diffusion may enhance edges [21]. After some time this filter creates segmentation-like results which are piecewise almost constant. For $t \rightarrow \infty$, however, the image becomes completely flat [4]. Well-posedness results for this filter can be found in $[5,4]$ and a scale-space interpretation in terms of an extremum principle as well as decreasing variance, decreasing energy, and increasing entropy is given in [4].

The effect of this diffusion filter is illustrated in Figure 1 (c),(d). We observe that it creates piecewise almost constant regions that are separated by sharp edges. If the images are very noisy, however, the filter performance deteriorates near edges where it tends to preserve these noisy structures by decreasing the diffusivity. In the next scetion we are concerned with a related method that is better suited for noise elimination than isotropic nonlinear diffusion filtering.

\subsection{Variational Image Restoration}

Many variational methods for image restoration (such as $[6,7,22]$ ) obtain a filtered version of some degraded image $f$ as the minimizer of an energy functional of type

$$
E_{f}(u):=\int_{\Omega}\left((u-f)^{2}+\alpha \Psi\left(|\nabla u|^{2}\right)\right) d x,
$$

where the regularizer $\Psi$ is an increasing function. The first summand encourages similarity between the restored image and the original one, while the second summand rewards smoothness. The smoothness weight $\alpha>0$ is called regularization parameter.

From variational calculus it follows that the minimizer of $E_{f}(u)$ satisfies the so-called Euler-Lagrange equation

$$
0=\operatorname{div}\left(\Psi^{\prime}\left(|\nabla u|^{2}\right) \nabla u\right)+\frac{1}{\alpha}(f-u)
$$

This can be regarded as the steady-state $(t \rightarrow \infty)$ of the diffusion-reaction process

$$
\partial_{t} u=\operatorname{div}\left(\Psi^{\prime}\left(|\nabla u|^{2}\right) \nabla u\right)+\frac{1}{\alpha}(f-u)
$$





Figure 1: (a) Top Left: test image. (b) Top Right: Gaussian noise with zero mean added. (c) Middle Left: nonlinear diffusion filtering of (a). (d) Middle Right: nonlinear diffusion filtering of (b). (e) Bottom Left: variational restoration of (a). (f) Bottom Right: variational restoration of $(b)$. 
This shows the close connection between variational image restoration and diffusion filtering. Indeed, much more relations have been discovered recently; see [24] for more details. In our case the convex potential [23]

$$
\Psi\left(|\nabla u|^{2}\right)=\lambda \sqrt{1+|\nabla u|^{2} / \lambda^{2}}+\varepsilon|\nabla u|^{2} \quad(\lambda, \varepsilon>0)
$$

is used. The corresponding diffusivity in (9) is given by its derivative

$$
\Psi^{\prime}\left(|\nabla u|^{2}\right)=\frac{1}{\sqrt{1+|\nabla u|^{2} / \lambda^{2}}}+\varepsilon
$$

Choosing a potential function $\Psi\left(s^{2}\right)$ that is convex in $s$ allows to guarantee wellposedness and stable algorithms [6]. For nonconvex potentials as in [25, 26], several well-posedness questions are open. Moreover, the diffusion-reaction equation (2.2) converges globally (i.e. for all initial values) to the solution of the Euler-Lagrange equation (8). It should be noted that the convex potential implies that the corresponding diffusive flux $\Phi(s)=s \Psi^{\prime}\left(s^{2}\right)$ is increasing in $s$. Thus, backward diffusion does not appear and edge enhancement is not possible. Nevertheless, since the diffusivity $\Psi^{\prime}\left(|\nabla u|^{2}\right)$ is decreasing in $|\nabla u|^{2}$, smoothing at edges is reduced and discontinuities are better preserved than in linear smoothing methods.

Figure 1(e),(f) depicts the filter performance of this method. It has a remarkable robustness under noise, but, in contrast to the nonlinear diffusion filter of Catté et al., it cannot enhance edges. This situation can be handled by more sophisticated diffusion filters such as edge-enhancing anisotropic diffusion [27]. They, however, require more complicated numerical algorithms that are beyond the scope of the present paper.

\section{Efficient Algorithms for PDE-Based Regulariza- tion}

\subsection{Limitations of Conventional Schemes}

Let us first consider finite difference approximations to the $m$-dimensional diffusion filter of Catté et al..

A discrete $m$-dimensional image can be regarded as a vector $f \in \mathbb{R}^{N}$, whose components $f_{i}, i \in\{1, \ldots, N\}$ display the grey values at the pixels. Pixel $i$ represents the location $x_{i}$. Let $h_{l}$ denote the grid size in the $l$ direction. We consider discrete times $t_{k}:=k \tau$, where $k \in \mathbb{N}_{0}$ and $\tau$ is the time step size. By $u_{i}^{k}$ and $g_{i}^{k}$ we denote approximations to $u\left(x_{i}, t_{k}\right)$ and $g\left(\left|\nabla u_{\sigma}\left(x_{i}, t_{k}\right)\right|^{2}\right)$, respectively, where the gradient is replaced by central differences.

The simplest discretization of the diffusion equation with reflecting boundary conditions is given by

$$
\frac{u_{i}^{k+1}-u_{i}^{k}}{\tau}=\sum_{l=1}^{m} \sum_{j \in \mathcal{N}_{l}(i)} \frac{g_{j}^{k}+g_{i}^{k}}{2 h_{l}^{2}}\left(u_{j}^{k}-u_{i}^{k}\right) .
$$


where $\mathcal{N}_{l}(i)$ consists of the two neighbors of pixel $i$ along the $l$ direction (boundary pixels may have only one neighbor). In vector-matrix notation this becomes

$$
\frac{u^{k+1}-u^{k}}{\tau}=\sum_{l=1}^{m} A_{l}\left(u^{k}\right) u^{k} .
$$

$A_{l}$ describes the diffusive interaction in $l$ direction. One can calculate $u^{k+1}$ directly (explicitly) from $u^{k}$ without any matrix inversion:

$$
u^{k+1}=\left(I+\tau \sum_{l=1}^{m} A_{l}\left(u^{k}\right)\right) u^{k} .
$$

For this reason it is called explicit scheme. Each explicit iteration step can be performed very fast, but the step size has to be very small: one can show [8] that in order to guarantee stability, the step size must satisfy

$$
\tau \leq \frac{1}{\sum_{l=1}^{m} \frac{2}{h_{l}^{2}}}
$$

For most practical applications, this restriction requires to use a very high number of iterations, such that the explicit scheme is rather slow.

Thus, we consider a slightly more complicated discretization next, namely

$$
\frac{u^{k+1}-u^{k}}{\tau}=\sum_{l=1}^{m} A_{l}\left(u^{k}\right) u^{k+1} .
$$

This scheme does not give the solution $u^{k+1}$ directly (explicitly): It requires to solve a linear system first. It is called a linear-implicit (semi-implicit) scheme. The solution $u^{k+1}$ is given by

$$
u^{k+1}=\left(I-\tau \sum_{l=1}^{m} A_{l}\left(u^{k}\right)\right)^{-1} u^{k} .
$$

This scheme is absolutely stable [4].

In the 1-D case the system matrix is tridiagonal and diagonally dominant. For such a system a Gaussian algorithm for tridiagonal systems (also called Thomas algorithm) solves the problem in linear complexity [8].

For dimensions $m \geq 2$, however, it is not possible to order the pixels in such a way that in the $i$-th row all nonvanishing elements of the system matrix can be found within the positions $[i, i-m]$ to $[i, i+m]$ : Usually, the matrix reveals a much larger bandwidth. Applying direct algorithms such as Gaussian elimination would destroy the zeros within the band and would lead to an immense storage and computation effort. Typical iterative algorithms become slow for large $\tau$, since this increases the condition number of the system matrix. Thus, in spite of its absolute stability, the semi-implicit scheme is often not much faster than the explicit one. 


\subsection{AOS Schemes}

In order to address the preceding problem we consider a modification of (15), namely the additive operator splitting (AOS) scheme [8]

$$
u^{k+1}=\frac{1}{m} \sum_{l=1}^{m}\left(I-m \tau A_{l}\left(u^{k}\right)\right)^{-1} u^{k} .
$$

The operators $B_{l}\left(u^{k}\right):=I-m \tau A_{l}\left(u^{k}\right)$ describe one-dimensional diffusion processes along the $x_{l}$ axes. Under a consecutive pixel numbering along the direction $l$ they come down to strictly diagonally dominant tridiagonal matrices which can be efficiently inverted by the Thomas algorithm.

Moreover, (16) has the same first-order Taylor expansion in $\tau$ as the explicit and semiimplicit scheme: all methods are $O\left(\tau+h_{1}^{2}+\ldots+h_{m}^{2}\right)$ approximations to the continuous equation.

Since it is an additive splitting, all coordinate axes are treated in exactly the same manner. This is in contrast to conventional splitting techniques from the literature, which are multiplicative [28]. They may produce different results if the image is rotated by 90 degrees.

Recently a general framework for discrete nonlinear diffusion scale-spaces has been discovered, which guarantees that the discretization reveals the same scale-space properties as its continuous counterpart [4]. One can|verify [8] that the AOS scheme creates such a discrete nonlinear diffusion scale-space for every (!) step size $\tau$. As a consequence, it preserves the average grey level $\mu$, satisfies a causality property in terms of a maximum-minimum principle, and converges to a constant steady state. Moreover, the process is a simplifying, information-reducing transform with respect to many aspects: The $p$-norms

$$
\left\|u^{k}\right\|_{p}:=\left(\sum_{i=1}^{N}\left|u_{i}^{k}\right|^{p}\right)^{1 / p}
$$

and all even central moments

$$
M_{2 n}\left[u^{k}\right]:=\frac{1}{N} \sum_{j=1}^{N}\left(u_{j}^{k}-\mu\right)^{2 n}
$$

are decreasing in $k$, and the entropy

$$
S\left[u^{k}\right]:=-\sum_{j=1}^{N} u_{j}^{k} \ln u_{j}^{k},
$$

a measure of uncertainty and missing information, is increasing in $k$ (if $f_{j}$ is positive for all $j$ ).

Table 1 summarizes the features of the discussed schemes. Full algorithmic details of AOS schemes can be found in [8], and a parallel implementation for processing 3-D images is described in [29]. 
Table 1: Schemes which create discrete nonlinear diffusion scale-spaces.

\begin{tabular}{|l|l|c|c|c|}
\hline scheme & formula & stability & costs/iter. & efficiency \\
\hline explicit & $u^{k+1}=\left(I+\tau \sum_{l=1}^{m} A_{l}\left(u^{k}\right)\right) u^{k}$ & $\tau \leq \frac{1}{\sum_{l=1}^{m} \frac{2}{h_{l}^{2}}}$ & very low & low \\
\hline semi-implicit & $u^{k+1}=\left(I-\tau \sum_{l=1}^{m} A_{l}\left(u^{k}\right)\right)^{-1} u^{k}$ & $\tau<\infty$ & high & fair \\
\hline AOS & $u^{k+1}=\frac{1}{m} \sum_{l=1}^{m}\left(I-m \tau A_{l}\left(u^{k}\right)\right)^{-1} u^{k}$ & $\tau<\infty$ & low & high \\
\hline
\end{tabular}

Many nonlinear diffusion problems require only the elimination of noise and some small-scale details. Often this can be accomplished with no more than 5 iterations in sufficient precision. We shall see that this takes about half a second for a $256^{2}$ image on current PCs or workstations.

\subsection{Pyramid AOS}

Let us now investigate a novel extension of the AOS framework to the variational image restoration method. In matrix-vector notation a semi-implicit discretization of (9) is given by

$$
\frac{u^{k+1}-u^{k}}{\tau}=\sum_{l=1}^{m} A_{l}\left(u^{k}\right) u^{k+1}+\alpha\left(f-u^{k+1}\right) .
$$

Solving for $u^{k+1}$ yields

$$
u^{k+1}=\left(I-\frac{\tau}{1+\frac{\tau}{\alpha}} \sum_{l=1}^{m} A_{l}\left(u^{k}\right)\right)^{-1} \frac{u^{k}+\frac{\tau}{\alpha} f}{1+\frac{\tau}{\alpha}} .
$$

In analogy to the previous section we may approximate this scheme by its AOS variant

$$
u^{k+1}=\frac{1}{m} \sum_{l=1}^{m}\left(I-\frac{m \tau}{1+\frac{\tau}{\alpha}} A_{l}\left(u^{k}\right)\right)^{-1} \frac{u^{k}+\frac{\tau}{\alpha} f}{1+\frac{\tau}{\alpha}}
$$

which again comes down to recursive filtering.

In contrast to the pure diffusion filter, however, we are now interested in approximating the steady-state solution for $t \rightarrow \infty$. Even with large time step sizes, the diffusion process will mainly act within a fairly small vicinity around each pixel. Thus, many iterations are required if the image size is large. In order to speed up the process, we may embed the AOS scheme into a pyramid framework. The idea is as follows:

- create a Gaussian pyramid decomposition [30] with the smoothing mask $\left(\frac{1}{4}, \frac{1}{2}, \frac{1}{4}\right)$ 
- adapt the filter parameters to the downsampled image. The scaling behaviour of diffusion-reaction processes requires that $\alpha$ must be divided by 4 if one reduces the image size by a factor 2 . Since the smoothing mask $\left(\frac{1}{4}, \frac{1}{2}, \frac{1}{4}\right)$ reduces the contrast of an ideal step edge by $25 \%$, it follows that the contrast parameter $\lambda$ has to be multiplied by 0.75 .

- start with the coarsest level (a $2 \times 2$ image), and apply a specified number of AOS iterations.

- expand this solution to the next finer level by linear interpolation, and use it as initial value for AOS iterations at this level.

- proceed in the same way until convergence at the finest level is reached.

Figure 2 illustrates the effect of pyramid AOS. Typically, five iterations are sufficient in order to obtain good convergence at each level. Since the Gaussian pyramid decomposition can be performed with linear complexity, the overall complexity remains linear as well. We shall see that regularizing a $256^{2}$ image on a current PC or workstation with this pyramid AOS scheme requires only around 0.5 CPU seconds.

It should be noted that the pyramid embedding converges to the same regularized image than pure AOS iterations would do, since the convex variational approach is globally convergent. However, pyramid AOS converges faster because of its better initial data that are provided by the previous pyramid level.

\section{Watershed Segmentation with Region Merging}

The preceding PDE-based regularization techniques lead to simplified images where noise and unimportant fine-scale details have been removed.

In order to create a true segmentation, we have to postprocess the regularized image by a technique which gives an edge map without dangling edges. This edge map should lead to a partitioning of the entire image into a finite number of regions, it should handle the semantically important corners and junctions gracefully, and - last but not least it should be fast. Classical gradient-based edge detectors such as a Sobel operator or Kirsch masks are not sufficient for this task, as they do not give closed contours. This also holds for more sophisticated variants such as the Canny edge detector [31].

We found a watershed technique $[32,33]$ based on the squared gradient magnitude very useful for these purposes. Such a technique regards an image as a landscape where the intensity values correspond to the elevation. Areas where a rain drop would drain to the same minimum are denoted as catchment basins, and the lines separating adjacent catchment basins are called watersheds. Watersheds are a morphological technique, since they are invariant under monotone grey scale-transformations. They lead to an image segmentation into regions, and they can describe edge junctions [35]. This is in contrast to edge detectors based on zero-crossings of differential operators such as the Laplacian-of-Gaussian [34]: they do not allow to detect T-junctions [36]. 

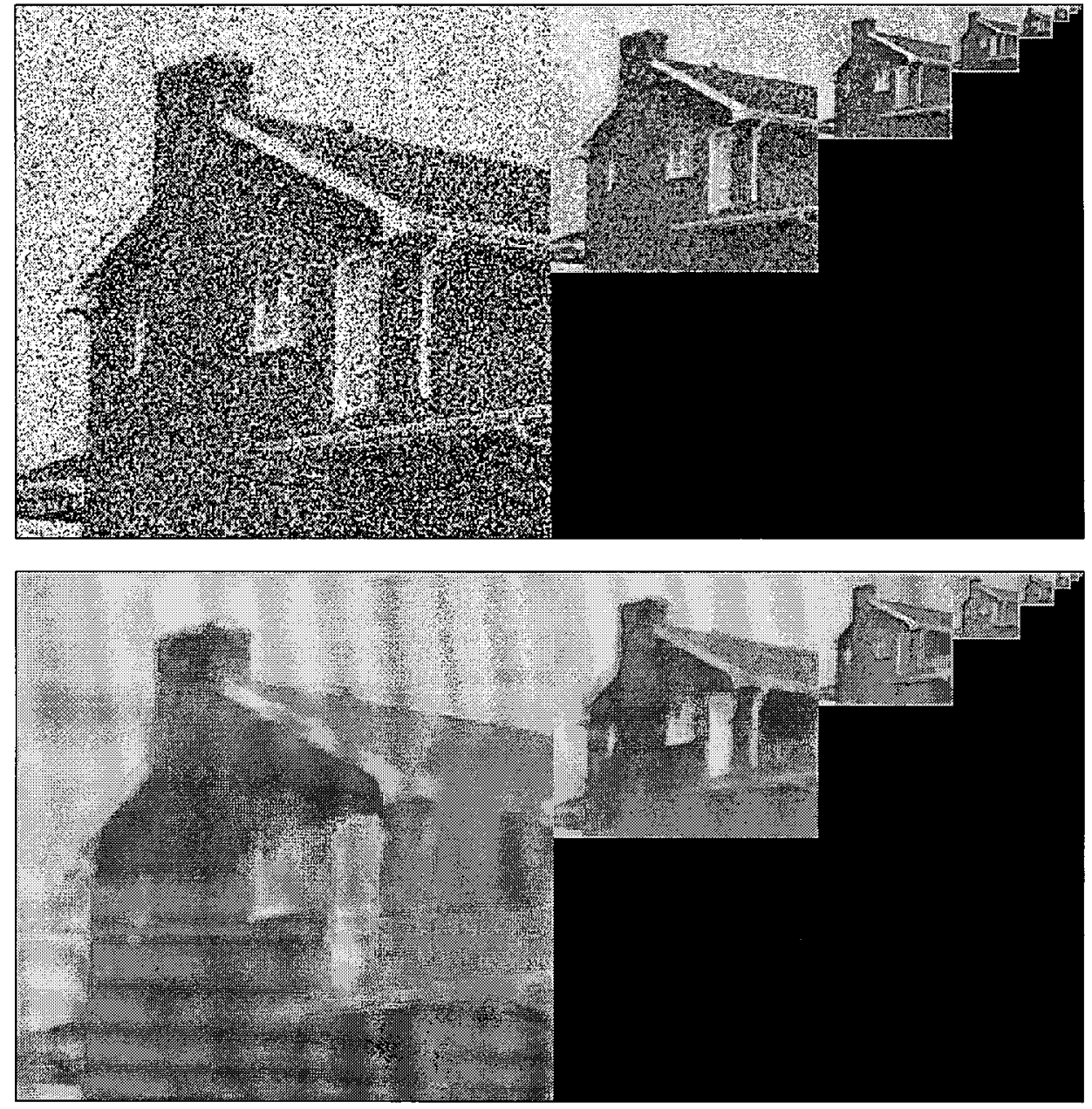

Figure 2: (a) Top: Gaussian pyramid of a noisy test image. (b) Bottom: regularized by pyramid AOS. 
We use Fairfield's watershed algorithm [37]. Our code is based on an implementation of Oltmans [38], where the Pascal code has been transferred into $\mathrm{C}$ and minor modifications have been included in order to optimize its performance.

The basic idea of this algorithm is sliding downhill on the gradient squared surface until one arrives at a local minimum. Then one replaces all pixels along this path by the image intensity at its corresponding extremum. This algorithm has linear complexity. The squared image gradient is calculated by Sobel operators.

Watershed algorithms often create too many segments. Although this oversegmentation is less dominant in the PDE-regularized image than in the original one, it may still lead to problems. Numerous ways have been proposed in order to deal with the oversegmentation problem, for instance by using markers [33,39], region merging [40,41, 10], or scale-space techniques $[13,17,14]$. In our case we shall see that a simple region merging strategy is adequate.

In such a step, adjacent regions are merged if their contrast difference is below a specified threshold. This contrast parameter can be related to the contrast parameter $\lambda$ of the PDE-based regularization, thus it does not constitute an additional parameter. Finding a connected region where neighboring pixels do not differ by more than a specified contrast value can be performed in linear complexity and the result is independent of the order in which the algorithm runs through the pixels. Thus, the entire segmentation algorithm is invariant under image rotations by 90 degrees and it reveals a linear total complexity.

A watershed segmentation of a $256^{2}$ image with subsequent region merging takes about $0.2 \mathrm{CPU}$ seconds on a PC or workstation. Thus, the overall segmentation time including the PDE-based regularization is far less than 1 second.

\section{Experiments}

Figure 3 illustrates how preprocessing by nonlinear diffusion filtering greatly reduces the number of segments in a medical MR image: We also observe that under nonlinear diffusion the segment boundaries remain well located and need not be traced back in order to improve their localization. As can be seen for instance at the cerebellum, the segments correspond well with the depicted physical objects. Moreover, segmentation of elongated objects does not create any problem. For comparison purposes with a classic approach, Figure 3(e) shows the result of an edge detector based on Sobel operators. Here the gradient magnitude has to be postprocessed in order to give useful information. But even with sophisticated postprocessing strategies such as hysteresis thresholding and nonmaximum suppression, there remains one significant difference to a watershed segmentation: the contours are not closed. Hence such an operator does not give a partitioning of the image domain into segments. The latter one can be achieved by considering level sets of a differential operator such as the zero crossings of the Laplacian in Figure 3(f). In this case fairly large Gaussians are required in order to prevent oversegmentation. As a result, image structures become much more dislocated that in the watershed approach with nonlinear diffusion as preprocessing. This is clearly visible when comparing the contours of the cerebellum in Figs. 3(d) and (f). Another 
Table 2: CPU times for the different steps of the segmentation algorithm when $256^{2}$ images are processed.

\begin{tabular}{|l|l|c|}
\hline method & Sun Ultra 60 & PC (Pentium II MMX, 440 Mhz) \\
\hline $\begin{array}{l}\text { nonlinear diffusion } \\
(5 \text { iterations) }\end{array}$ & $0.494 \mathrm{~s}$ & $0.591 \mathrm{~s}$ \\
\hline $\begin{array}{l}\text { variational restoration } \\
(5 \text { iterations per level) }\end{array}$ & $0.407 \mathrm{~s}$ & $0.516 \mathrm{~s}$ \\
\hline $\begin{array}{l}\text { watershed transformation } \\
\text { with region merging }\end{array}$ & $0.162 \mathrm{~s}$ & $0.199 \mathrm{~s}$ \\
\hline
\end{tabular}

difference between these two approaches consists of the behavior at junctions: as already mentioned and as is visible in Figure 3(f), zero crossings cannot meet at T-junctions, whereas watersheds do.

In Figure 4 it is shown that the merging step can be essential for avoiding the oversegmentation problems in the watershed algorithm. Nonlinear diffusion may create almost piecewise constant areas, but small fluctuations within such an area introduce many semantically irrelevant catchment basins. Such fluctuations can also be caused by quantization errors, e.g. by storing grey values in a bytewise manner. Merging adjacent regions with similar grey values constitutes a simple remedy for these problems.

Finally, Figure 5 gives a comparison between the two PDE-based regularization techniques. The results are in complete accordance with those from Figure 1 . The contrast-enhancing nonlinear diffusion method gives more realistic results for images with less noise, as can be seen from the segmentation of the arms and legs in Figure 5(c). However, in a more noisy situation, the quality of this method degrades significantly. The variational method that does not allow contrast enhancement, on the other hand, does not reach the qualities of nonlinear diffusion preprocessing (Fig. 5(e)), but is very stable under noise (Fig. 5(f)). It is thus the better preprocessing method for noisy images. Again it should be emphasized that there exist more sophisticated nonlinear diffusion methods that combine the advantages of both approaches studied here [27]. Their efficient algorithmic realization, however, is more complicated and requires further research.

Table 2 presents precise CPU times for our segmentation algorithm both for a workstation (Sun Ultra 60) and a PC (Pentium II MMX, $440 \mathrm{Mhz}$ ), when $256^{2}$ images are processed and a GNU C compiler is used. On both architectures, preprocessing by means of nonlinear diffusion or variational restoration can be achieved in about 0.5 seconds, while the watershed transformation with region merging takes 0.2 seconds. This shows that the complete algorithm allows to segment $256^{2}$ images in much less than a second. With a PC with $700 \mathrm{MHz}$ it is even possible to segment two such images in less than one second. Moreover, it should be taken into account that the AOS algorithm, which is the most time consuming subroutine of the entire method, is very well-suited for parallel computing [29]. Therefore, further speed up can be achieved in a straightforward way. 

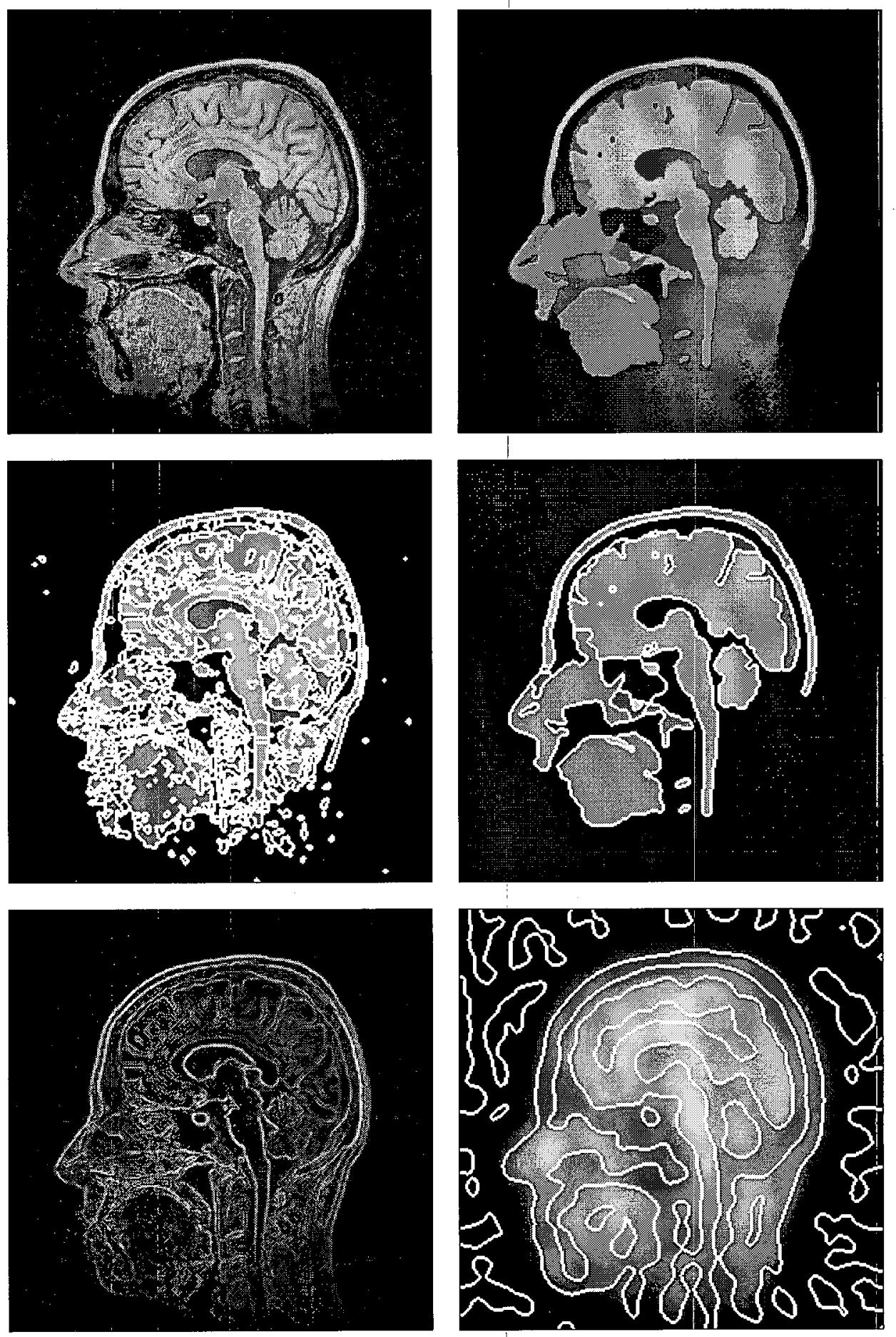

Figure 3: (a) Top Left: MR image. (b) Top Right: nonlinear diffusion filtering of (a). (c) Middle Left: segmentation of (a). (d) Middle Right: segmentation of (b). (e) Bottom Left: Sobel operator applied to (a). (f) Bottom Right: Zero crossings of the Laplacian-of-Gaussian of (a). 

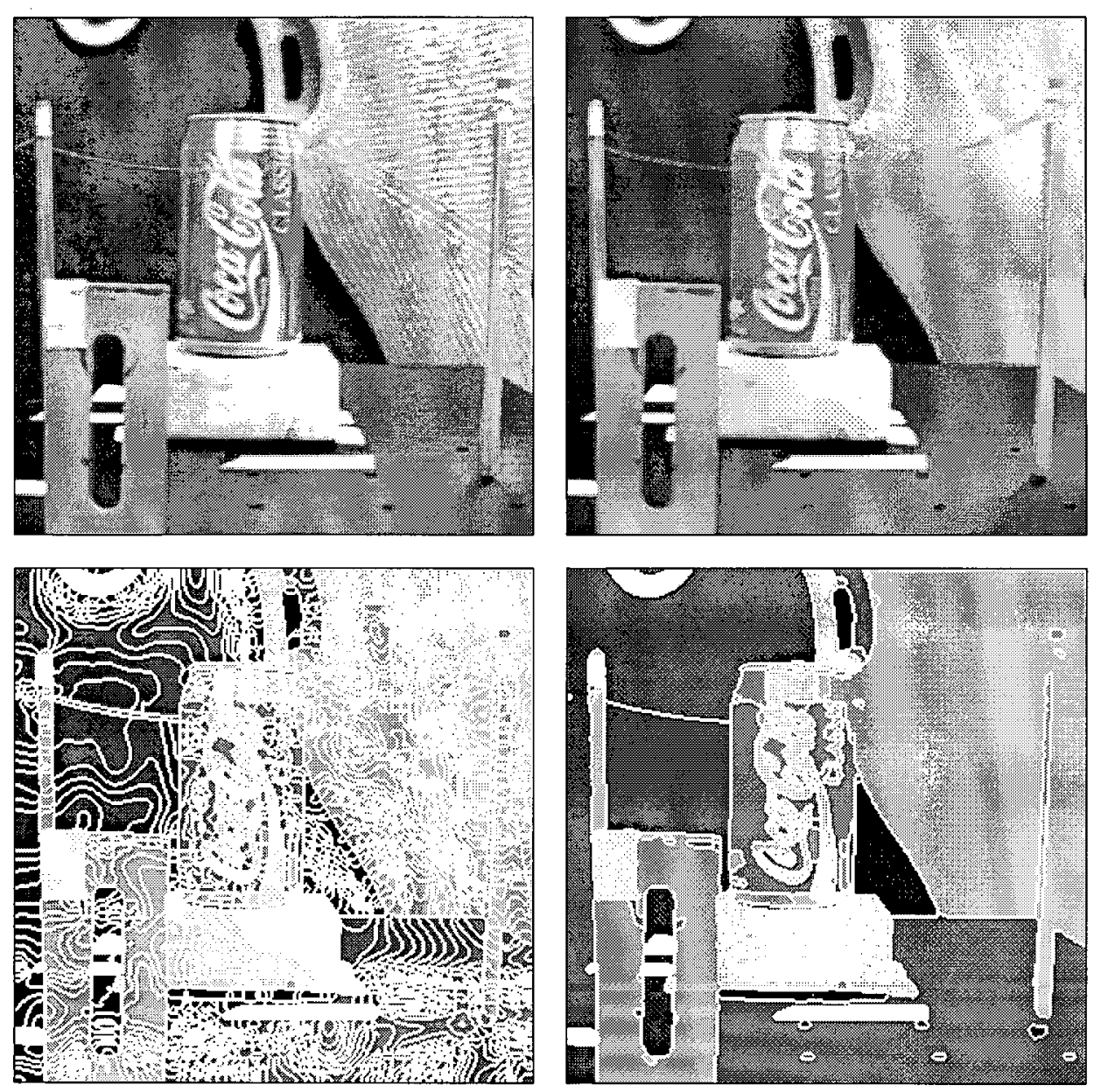

Figure 4: (a) Top Left: test image. (b) Top Right: nonlinear diffusion filtering of (a). (c) Bottom Left: segmentation of (b) without merging. (d) Bottom Right: segmentation of (b) with merging. 

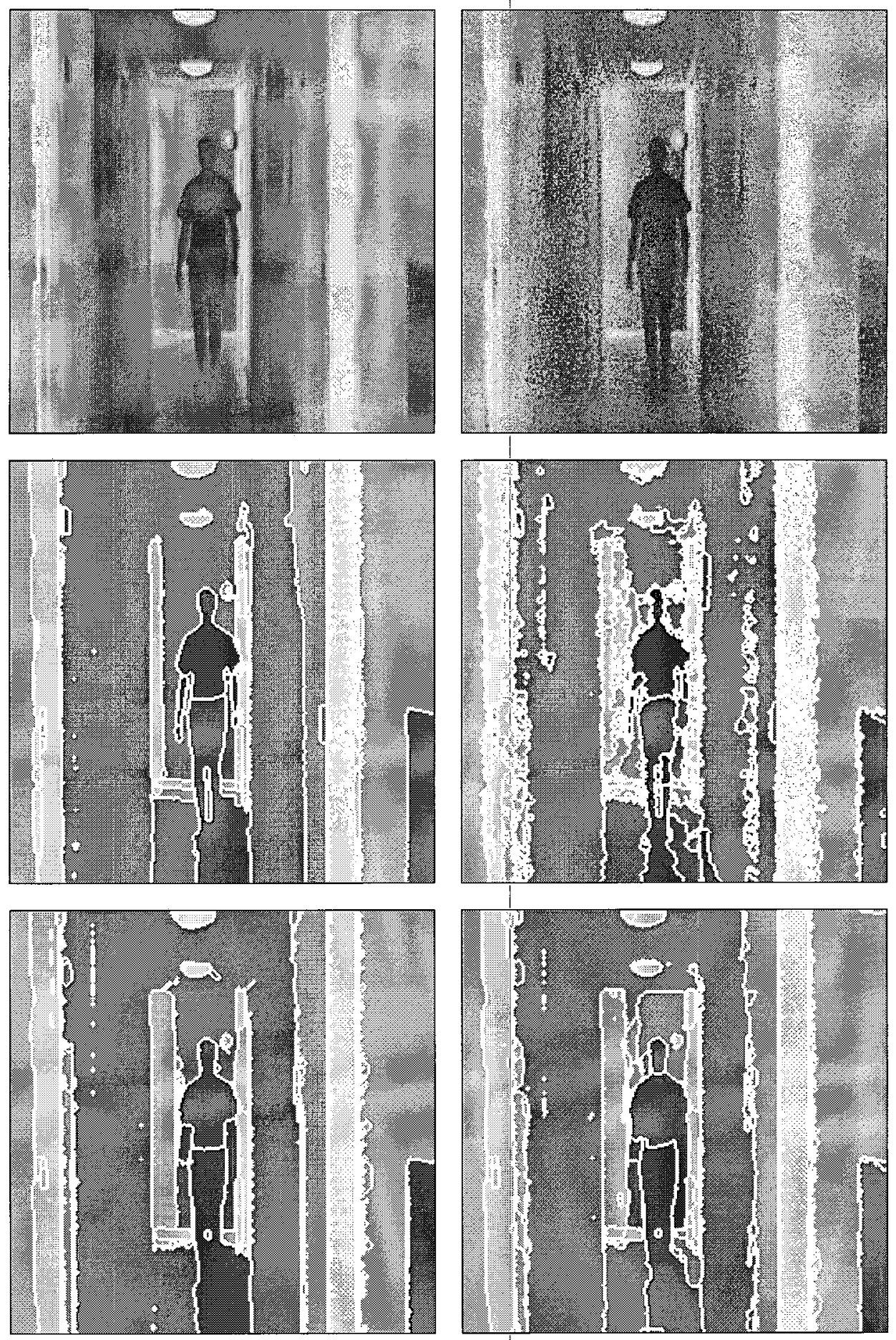

Figure 5: (a) Top Left: hallway scene. (b) Top Right: Gaussian noise added. (c) Middle Left: segmentation of (a) with nonlinear diffusion as preprocessing. (d) Middle Right: segmentation of (b) with nonlinear diffusion as preprocessing. (e) Bottom Left: segmentation of (a) with variational restoration as preprocessing. (f) Bottom Right: segmentation of (b) with variational 


\section{Summary}

We have presented efficient algorithms for two prototypes of PDE-based regularization techniques. These regularizations simplify subsequent segmentation tasks significantly, such that already a simple watershed algorithm with region merging gives good results. These segmentation techniques are very fast thanks to the use of AOS schemes and a novel pyramid AOS algorithm. This makes them attractive for many time-critical applications. All axes are treated equally, since the result is independent of the pixel ordering. The entire algorithm can be extended in a straightforward way to $m$-dimensional data, and the linear complexity in the pixel number remains valid in any dimension.

Acknowledgements. This work has been carried out within the EU-TMR network VIRGO. The author thanks Ole Fogh Olsen and Mads Nielsen for the hallway image, and Hans Oltmans for providing his implementation of Fairfield's watershed algorithm.

\section{References}

[1] V. Caselles, J.M. Morel, G. Sapiro, A. Tannenbaum (Eds.), Special issue on partial differential equations and geometry-driven diffusion in image processing and analysis, IEEE Trans. Image Proc, Vol. 7, No. 3, March 1998.

[2] B. ter Haar Romeny, L. Florack, J. Koenderink, M. Viergever (Eds.), Scale-space theory in computer vision, Lecture Notes in Computer Science, Vol. 1252, Springer, Berlin, 1997.

[3] M. Nielsen, P. Johansen, O.F. Olsen, J. Weickert (Eds.), Scale-space theories in computer vision, Lecture Notes in Computer Science, Vol. 1682, Springer, Berlin, 1999.

[4] J. Weickert, Anisotropic diffusion in image processing, ECMI Series, TeubnerVerlag, Stuttgart, 1998.

[5] F. Catté, P.L. Lions, J.M. Morel, T. Coll, Image selective smoothing and edge detection by nonlinear diffusion, SIAM J. Numer. Anal., Vol. 29, 182-193, 1992.

[6] C. Schnörr, Unique reconstruction of piecewise smooth images by minimizing strictly convex non-quadratic functionals, J. Math. Imag. Vision, Vol. 4, 189-198, 1994.

[7] P. Charbonnier, L. Blanc-Féraud, G. Aubert, M. Barlaud, Two deterministic halfquadratic regularization algorithms for computed imaging, Proc. IEEE Int. Conf. Image Processing (ICIP-94, Austin, Nov. 13-16, 1994), Vol. 2, IEEE Computer Society Press, Los Alamitos, 168-172, 1994.

[8] J. Weickert, B.M. ter Haar Romeny, M.A. Viergever, Efficient and reliable schemes for nonlinear diffusion filtering, IEEE Trans. Image Proc., Vol. 7, 398-410, 1998.

[9] S.T. Acton, Multigrid anisotropic diffusion, IEEE Trans. Image Proc., Vol. 7, 280291, 1998. 
[10] S.C. Orphanoudakis, G. Tziritas, K. Haris, A hybrid algorithm for the segmentation of $2 D$ and $3 D$ medical images, Y. Bizais, C. Barillot, R. Di Paola (Eds.), Information processing in medical imaging, Kluwer, Dordrecht, 385-386, 1995.

[11] D. De Vleeschauer, F.A. Cheikh, R. Hamila, M. Gabbouj, Watershed segmentation of an image enhanced by Teager energy driven diffusion, Proc. Sixth Int. Conf. on Image Processing and its Applications (IPA 97, Dublin, July 15-17, 1997), 254-258, 1997.

[12] J. Sijbers, P. Scheunders, M. Verhoye, A. Van der Linden, D. Van Dyck, E. Raman, Watershed-based segmentation of $3 D M R$ data for volume quantization, Magnetic Resonance Imaging, Vol. 15, 679-688, 1997.

[13] L.D. Griffin, A.C.F. Colchester, G.P. Robinson, Scale and segmentation of greylevel images using maximum gradient paths, Image and Vision Computing, Vol. 10, 389-402, 1992.

[14] O.F. Olsen, Multiscale watershed segmentation, J. Sporring, M. Nielsen, L. Florack, P. Johansen (Eds.), Gaussian scale-space theory, Kluwer, Dordrecht, 191-200, 1997.

[15] M. Sramek, T. Wrbka, Watershed based image segmentation - an effective tool for detecting landscape structure, E. Wenger, L.I. Dimitrov (Eds.), Sixth International Workshop on Digital Image Processing and Computer Graphics: Applications in Humanities and Natural Sciences, SPIE Vol. 3346, 227-235, 1997.

[16] O.F. Olsen, J. Sporring, Segmenting by compression using linear scale-space and the watersheds, M. Nielsen, P. Johansen, O.F. Olsen, J. Weickert (Eds.), Scalespace theories in computer vision, Lecture Notes in Computer Science, Vol. 1682, Springer, Berlin, 513-518, 1999.

[17] P.T. Jackway, Gradient watersheds in morphological scale-space, IEEE Trans. Image Proc., Vol. 5, 913-921, 1996.

[18] W.J. Niessen, K.L. Vincken, J. Weickert, M.A. Viergever, Nonlinear multiscale representations for image segmentation, Computer Vision and Image Understanding, Vol. 66, 233-245, 1997.

[19] W.J. Niessen, K.L. Vincken, J. Weickert, B:M. ter Haar Romeny, M.A. Viergever, Multiscale segmentation of three-dimensional MR brain images, Int. J. Comput. Vision, Vol. 31, May 1999.

[20] J. Weickert, Fast segmentation methods based on partial differential equations and the watershed transformation, P. Levi, R.-J. Ahlers, F. May, M. Schanz (Eds.), Mustererkennung 1998, Springer, Berlin, 93-100, 1998.

[21] P. Perona, J. Malik, Scale space and edge detection using anisotropic diffusion, IEEE Trans. Pattern Anal. Mach. Intell., Vol. 12, 629-639, 1990. 
[22] R. Deriche, O. Faugeras, Les EDP en traitement des images et vision par ordinateur, Traitement du Signal, Vol. 13, No. 6, 1996.

[23] M.Z. Nashed, O. Scherzer, Least squares and bounded variation regularization with nondifferentiable functionals, Numerical Functional Analysis and Optimization, Vol. 19, 873-901, 1998.

[24] O. Scherzer, J. Weickert, Relations between regularization and diffusion filtering, J. Math. Imag. Vision, Vol. 12, 43-63, 2000.

[25] N. Nordström, Biased anisotropic diffusion - a unified regularization and diffusion approach to edge detection, Image and Vision Computing, Vol. 8, 318-327, 1990.

[26] D. Geiger, A. Yuille, A common framework for image segmentation, Int. J. Comput. Vision, Vol. 6, 227-243, 1991.

[27] J. Weickert, Theoretical foundations of anisotropic diffusion in image processing, Computing, Suppl. 11, 221-236, 1996.

[28] G.I. Marchuk, Splitting and alternating direction methods, P.G. Ciarlet, J.-L. Lions (Eds.), Handbook of numerical analysis, Vol. I, 197-462, North Holland, Amsterdam, 1990.

[29] J. Weickert, J. Heers, C. Schnörr, K.J. Zuiderveld, O. Scherzer, H.S. Stiehl, Fast parallel algorithms for a broad class of nonlinear variational diffusion approaches, Real-Time Imaging, to appear.

[30] P.J. Burt, E.H. Adelson, The Laplacian pyramid as a compact image code, IEEE Trans. Comm., Vol. 31, 532-540, 1983.

[31] J. Canny, A computational approach to edge detection, IEEE Trans. Pattern Anal. Mach. Intell., Vol. 8, 679-698, 1986.

[32] S. Beucher, C. Lantuéjoul, Use of watersheds in contour detection, Proc. Int. Workshop on Image Processing, Real-Time Edge and Motion Detection/Estimation (Rennes, Sept. 17--21, 1979), IRISA Report No. 131, 2.1-2.12, 1979.

[33] F. Meyer, S. Beucher, Morphological segmentation, J. Vis. Comm. Image Repr., Vol. 1, 21-46, 1990.

[34] D. Marr, E. Hildreth, Theory of edge detection, Proc. R. Soc. London Ser. B, Vol. 207, 187-217, 1980 .

[35] L. Najman, M. Schmitt, Watershed of a continuous function, Signal Processing, Vol. 38, 99-112, 1994.

[36] V. Torre, T.A. Poggio, On edge detection, IEEE Trans. Pattern Anal. Mach. Intell., Vol. 8, 148-163, 1986. 
[37] J. Fairfield, Toboggan contrast enhancement for contrast segmentation, Proc. 10th Int. Conf. Pattern Recognition (ICPR 10, Atlantic City, June 16-21, 1990), IEEE Computer Society Press, Los Alamitos, Vol. 1, 712-716, 1990.

[38] H. Oltmans, A watershed algorithm, project work, Laboratory of Technomathematics, University of Kaiserslautern, Germany, 1993.

[39] L. Najman, M. Schmitt, Geodesic saliency of watershed contours and hierarchical segmentation, IEEE Trans. Pattern Anal. Mach. Intell., Vol. 18, 1163-1173, 1996.

[40] L. Vincent, P. Soille, Watersheds in digital spaces: An efficient algorithm based on immersion simulation, IEEE Trans. Pattern Anal. Mach. Intell., Vol. 13, 583-589, 1991.

[41] F. Maes, D. Vandermeulen, P. Suetens, G. Marchal, Computer-aided interactive object delineation using an intelligent paintbrush technique, N. Ayache (Ed.), Computer vision, virtual reality and robotics in medicine, Lecture Notes in Computer Science, Vol. 905, Springer, Berlin, 77-83, 1995. 